\title{
VLSI Implementation of an Efficient Lossless EEG Compression Design for Wireless Body Area Network
}

\author{
Chiung-An Chen ${ }^{1}$, Chen $\mathrm{Wu}^{2}$, Patricia Angela R. Abu ${ }^{3}$ and Shih-Lun Chen ${ }^{2, *}$ \\ 1 Department of Electrical Engineering, Ming Chi University of Technology, New Taipei City 243, Taiwan; \\ joannechen@mail.mcut.edu.tw \\ 2 Department of Electronic Engineering, Chung Yuan Christian University, Taoyuan City 320, Taiwan; \\ zac_wu@hotmail.com \\ 3 Department of Information Systems and Computer Science, Ateneo de Manila University, \\ Quezon City 1108, Philippines; pabu@ateneo.edu \\ * Correspondence: chischen@cycu.edu.tw; Tel.: +886-3-265-4610
}

Received: 15 July 2018; Accepted: 17 August 2018; Published: 28 August 2018

Featured Application: Wireless Body Sensor Network, Wireless Body Area Network, Remote Healthcare, Internet of Things and Wearable Device.

\begin{abstract}
Data transmission of electroencephalography (EEG) signals over Wireless Body Area Network (WBAN) is currently a widely used system that comes together with challenges in terms of efficiency and effectivity. In this study, an effective Very-Large-Scale Integration (VLSI) circuit design of lossless EEG compression circuit is proposed to increase both efficiency and effectivity of EEG signal transmission over WBAN. The proposed design was realized based on a novel lossless compression algorithm which consists of an adaptive fuzzy predictor, a voting-based scheme and a tri-stage entropy encoder. The tri-stage entropy encoder is composed of a two-stage Huffman and Golomb-Rice encoders with static coding table using basic comparator and multiplexer components. A pipelining technique was incorporated to enhance the performance of the proposed design. The proposed design was fabricated using a $0.18 \mu \mathrm{m}$ CMOS technology containing 8405 gates with $2.58 \mathrm{~mW}$ simulated power consumption under an operating condition of $100 \mathrm{MHz}$ clock speed. The CHB-MIT Scalp EEG Database was used to test the performance of the proposed technique in terms of compression rate which yielded an average value of 2.35 for 23 channels. Compared with previously proposed hardware-oriented lossless EEG compression designs, this work provided a $14.6 \%$ increase in compression rate with a $37.3 \%$ reduction in hardware cost while maintaining a low system complexity.
\end{abstract}

Keywords: EEG; lossless compression; VLSI architecture; wireless body area network

\section{Introduction}

The electroencephalogram (EEG) [1,2] signal has always been considered an inherent and crucial reference for the neurologist to diagnose any brain disorder. EEG is a technique used to record electrical activity generated by the human brain [3]. Other problems associated with the abnormal functioning of the brain which can be diagnosed by using EEG signals include coma, confusion, stroke, and tumors. Among various modalities available, ambulatory or portable EEG is expected to emerge as a potentially viable area for data compressions in EEG devices. This is attributed to the ease of accessibility and high patient comfort offered during the EEG signal acquisition procedure.

Traditional EEG monitors and recorders transmit EEG signals via cables. In recent years, the Wireless Body Sensor Network (WBSN) technology is being widely developed since it can greatly 
enhance the way people live in terms of comfort and convenience [4-6]. WBAN is also being developed due to its high potential to replace the use of batteries for portable devices [7-9]. Most portable wireless devices in the market, however, is limited to their onboard battery capacity as their main constraint for the long-term use of the device. One of the approaches to improve the efficiency of current wireless EEG recorders is making use of a lossless compression technique to reduce the power consumption during the transmission of EEG data.

In this paper, a novel dynamic voting prediction methodology is proposed in order to adaptively choose the optimal prediction function. In addition, a fuzzy decision unit is incorporated to improve the accuracy of prediction. A novel dynamic voting scheme is combined with the adaptive fuzzy prediction methodology for the EEG compression. Moreover, a novel tri-stage entropy encoding method is combined with the adaptive fuzzy and voting-based prediction. The comparators and multiplexer components were used to replace the complex computing components to achieve hardware sharing. A pipelining technique is used to improve the performance of this design. Simulation results showed that the proposed algorithm significantly improved the compression rate with a relatively low complexity in its VLSI implementation.

This paper is organized as follows: Section 2 presents a literature review of several lossless compression algorithms. Section 3 discusses the methodology of the proposed novel lossless EEG compression algorithm. This includes dynamic voting prediction, tri-stage entropy coding and its VLSI architecture. Section 4 lists and discusses the results of the proposed lossless EEG compression algorithm and its performance. A comparison with previous studies in terms of compression rate and VLSI design performance is also presented. Conclusions were summarized in Section 5.

\section{Literature Review}

A Chaotic Compressive Sensing (CCS) algorithm was proposed in [10] to solve both problems of energy saving and data security. A combined compression algorithm [4] provided different biomedical signals that showed a significant improvement in the compression ratio with a small maximum error for optimizing the network resource usage over Wireless Sensor Networks (WSN). A lossy compression algorithm based on online dictionaries provided a quantitative assessment for compression, reconstruction and energy consumption of wearable Internet of Things (IoT) was presented in [11].

Several varieties of EEG compression algorithms have been proposed in literature. Srinivasan et al. [12] proposed a high performance wavelet-based EEG compression while Sriraam et al. [13] presented an adaptive error modeling scheme. Shaw et al. [14] presented an excellent algorithm for EEG signals in high dimensionality. Shaw et al. proposed a compression method for multichannel EEG data with improved storage capacity and efficiency in transmission. A lossless and near-lossless algorithm of EEG compression was presented in [15]. The literature proposed two modes of compression for different platform with individual capability. An algorithm for classifying multichannel EEG data through a promising tool called the fuzzy multichannel EEG classifier was proposed in [16]. This supports different EEG signals that were collected at different time instants.

The previously proposed algorithms were able to attain a high performance in terms of both compression rate and complexity in their VLSI architecture. Chua et al. [17] proposed a VLSI implementation of a mixed bio-signal lossless data compression together with its EEG signal compression. The said work presented a detailed figure of merits for the different compression methodologies.

On the other hand, several compression techniques were also proposed for electrocardiography (ECG) signals. Chen et al. [18] developed a slope prediction based algorithm while Chen et al. [19] proposed a novel adaptive trending prediction with Huffman coding architecture that was extended by implementing a fuzzy decision technique for a more efficient and precise signal prediction [20]. These 3 previously proposed methods in literature provided a reliable reference for EEG compression in VLSI implementation. Recently, an adaptive resolution control [21] and a fuzzy resolution control [22] 
techniques for body signals have been proposed. Although these variable resolution control techniques can improve the compression rate efficiently, there is significant information that was lost in the body signal.

\section{Methodology}

\subsection{Lossless EEG Compression Algorithm}

The proposed novel lossless EEG compression algorithm consists of a predictor and an encoder. The result of the predictor is used as the input to the encoder. The predictor implements a voting-based scheme operating with the fuzzy decision technique that is designed to improve the efficiency of the entropy coding. The entropy encoder is a tri-stage design that involves pre-processing of data through the use of a two-stage Huffman encoder and a Golomb-Rice encoder. The following subsections discuss each of these components.

\subsubsection{Dynamic Voting Prediction}

Biomedical signals are by nature chaotic and fluctuate rapidly. As such, there is no single generic prediction function that is capable of accurately predicting a biomedical signal while maintaining a low system complexity. As opposed to designing several prediction algorithms for each and every kind of biomedical signal, this study proposes a dynamic voting prediction algorithm that is independent to the type of body signal in order to enhance the overall performance of the signal prediction. The dynamic voting prediction scheme proposed in this study is composed of the following steps: the first-stage prediction, second-stage prediction, and the voting prediction stage.

\section{First-Stage Prediction}

This preliminary step ensures that the data distribution will converge. This step made use of three ECG signal prediction functions that were proposed in previous studies namely linear, slope, and adaptive trending functions by Chen et al. [6], Chen et al. [18] and Chen et al. [23], respectively. These functions were simulated and tested to predict a given ECG signal. Table 1 lists the prediction functions and their respective compression rate on the ECG signals provided by the MIT-BIH arrhythmia database [24]. The adaptive trending function had the highest compression rate achieved.

Table 1. Compression rate on ECG signals of previously-proposed prediction functions.

\begin{tabular}{cc}
\hline Prediction Function & Compression Rate \\
\hline Linear [6] & 1.90 \\
Slope [18] & 2.38 \\
Adaptive Trending [20] & 2.56 \\
\hline
\end{tabular}

Second-Stage Prediction

The second-stage prediction involves a fuzzy decision component [20]. This step organizes the results of the first-stage prediction according to the two slope values diff_1 and diff_2 as shown in Figure 1. The values of diff_1 and diff_2 are equivalent to the absolute differences of $X(n-1)$ and $X(n-1)$, and $X(n-1)$ and $X(n-2)$, respectively. 


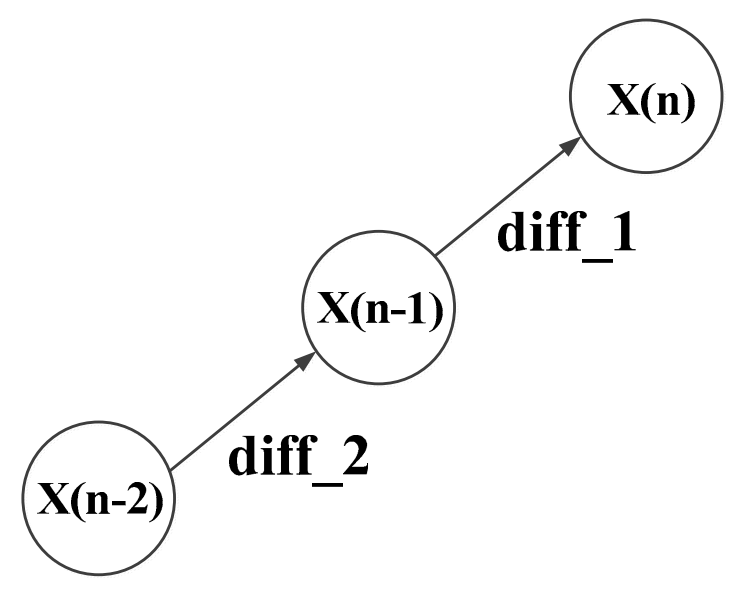

Figure 1. Slope prediction using diff_1 and diff_2.

Fuzzy Decision Rules

Figure 2 illustrates the fuzzy decision rule of the proposed EEG prediction methodology with threshold values of low, medium and high. The first-stage prediction outcomes are matched and picked by one of the six modules M1-M6 in the voting prediction stage. Given the compression rate of the different prediction functions in Table 1, the adaptive trending method shows higher performance than the linear and slope functions for ECG signal. Hence, the use of the fuzzy decision rule is selected as the base prediction scheme for the proposed prediction methodology for EEG signals.

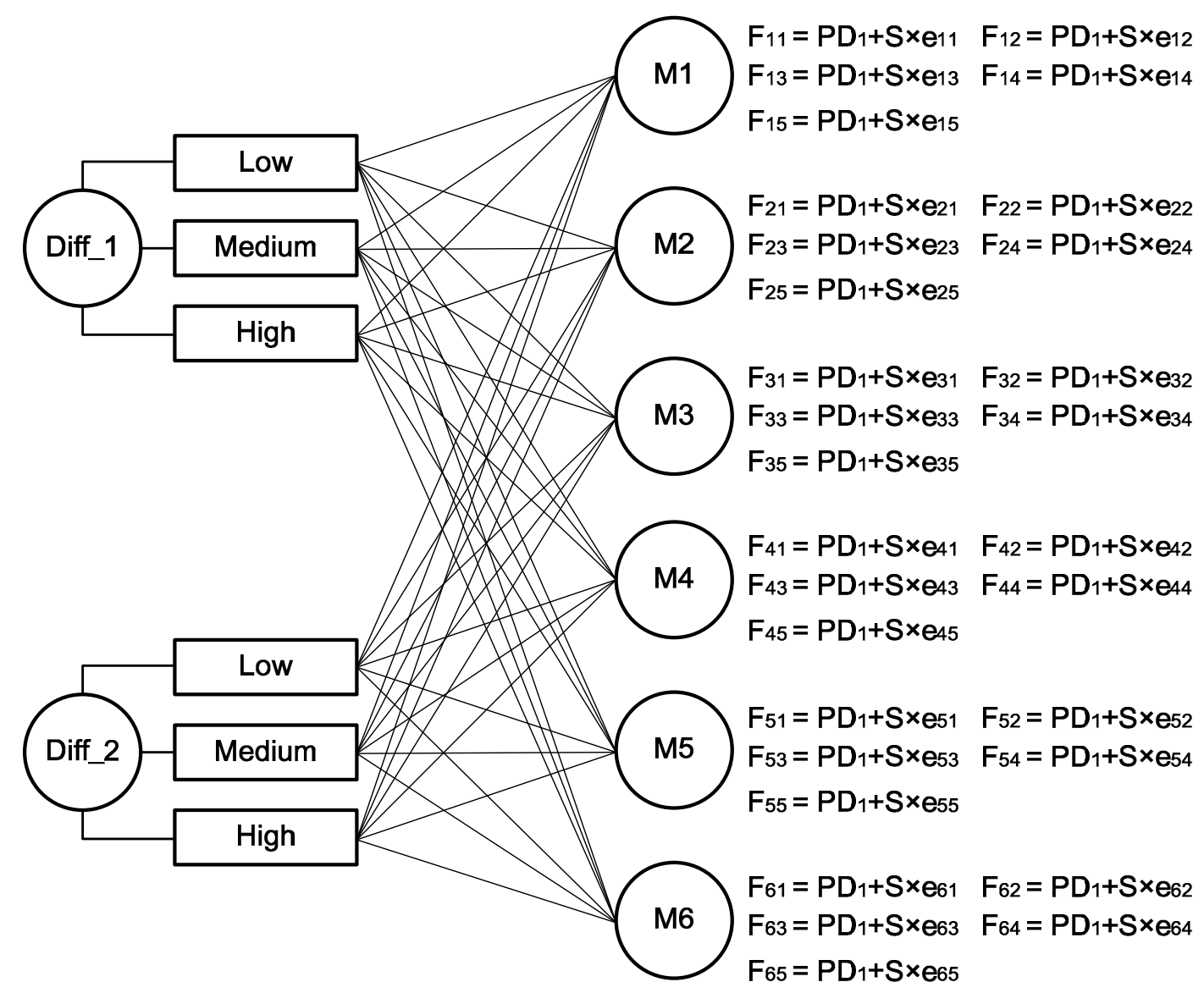

Figure 2. Fuzzy decision rules of the proposed prediction methodology for EEG signal. 
Dynamic Voting Scheme

The dynamic voting scheme component is developed to dynamically choose the optimal function during signal trend fluctuations. For every prediction session, one of the six modules will be activated to receive the data coming from the fuzzy decision module. Each of the modules contain five second-stage prediction functions, as shown in Figure 2. The following lists the constant and/or preset values: $e_{i j}$ is a constant where $1 \leq i \leq 6$ and $1 \leq j \leq 6$. Each fuzzy module contains 5 prediction equations, where each prediction equation has its own $e_{i j}$ value. $e_{i j}$ denotes the jth e value of the ith fuzzy module. A preset coefficient $e_{i j}$ value was used that is from the distribution of the difference value between the sampling points in the EEG dataset. The parameter $S$ is determined by getting the absolute value of diff_2 over diff_2. The number of votes for each function is monitored using five registers. The number of votes for each function is equal to the number of times that that particular function has the closest prediction among the five prediction functions. The function with the most number of votes will be executed with its resulting value stored in register $P D_{2}$ of the said voting prediction stage. The same procedure is performed for all the other four functions.

Figure 3 shows the dynamic voting procedure. $P D_{1}$ is the subtractor output with inputs $X(n)$ and $X(n-1)$. The upper flow shows that given $P D_{1}$, the optimal function is selected according to the highest number of votes where its value is to be stored in $P D_{2}$. The lower flow of data acquires the current result of the prediction module. The current prediction result will be used as a vote to change the numbers of the corresponding function and contribute to the next prediction session. Register $P D_{2}$ stores the resulting value of the function with the highest number of votes.

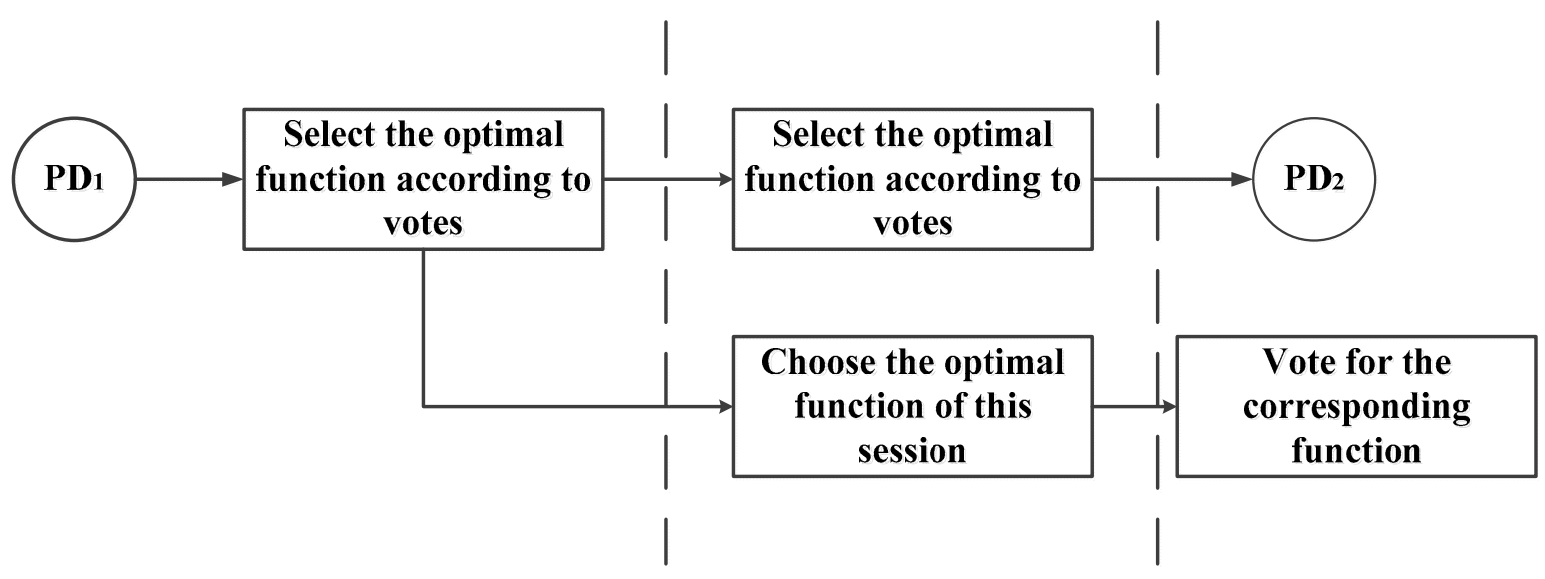

Figure 3. Dynamic voting process.

Figure 4 shows a sample prediction session. Figure 4a shows the result of each function together with the number of votes for each corresponding function. As can be seen, function 2 has the largest number of votes. Hence, the value stored in $P D_{2}$ register is chosen as the output of function 2 . However, in this session, function 1 has a resulting function value that is closest to -1 . As such, the optimal function is function 1 rather than function 2 . As such, the dynamic voting predictor will cast a vote to function 1 as shown in Figure $4 \mathrm{~b}$. 


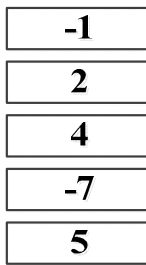

Results of each function

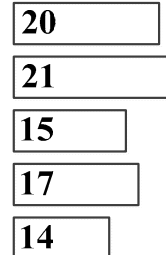

Number of votes of each function

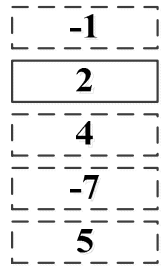

Result of

function 2

as $\mathrm{PD}_{2}$

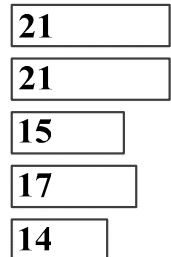

Number of

votes of each function

$a$

$b$

Figure 4. Sample data for one voting session: (a) result for each function, and (b) result for function 2.

\subsubsection{Tri-Stage Entropy Coding}

The entropy encoder incorporates both the Huffman coding and the Golomb-Rice coding algorithm. The Huffman coding encodes symbols that have higher probabilities with shorter code length according to the Huffman tree. However, as the value of the EEG signal data varies in a wide range, the Huffman tree would group up outstandingly. It is difficult to design a low-cost and a high performing VLSI architecture that has a block circuit and implements a tree with a very large depth. For this reason, a two-stage Huffman encoding technique [19] is applied to the data whose values have high probabilities. For the $P D_{2}$ values whose probabilities are relatively low, the absolute value is not too large. As such, the Golomb-Rice coding technique can efficiently shorten the length of the code by choosing an appropriate divider. This further enhances the performance of the Golomb-Rice coding. The $P D_{2}$ value can be transformed into a smaller value $P D^{\prime}(n)$ using Equation (1).

$$
\begin{gathered}
P D^{\prime}(n)=2 * P D_{2}(n)-2 k \text { if } P D_{2}(n) \geq 0 \\
P D^{\prime}(n)=2 * P D_{2}(n)-2 k+1 \text { if } P D_{2}(n)<0
\end{gathered}
$$

where $k$ is the first position in the Golomb-Rice table.

\subsection{VLSI Architecture}

Figure 5 shows the VLSI architecture of the proposed lossless EEG compression VLSI design. It illustrates its components which are composed of a dynamic voting predictor, a two-stage Huffman encoder, a Golomb-Rice encoder, pipeline registers, and their interconnections.

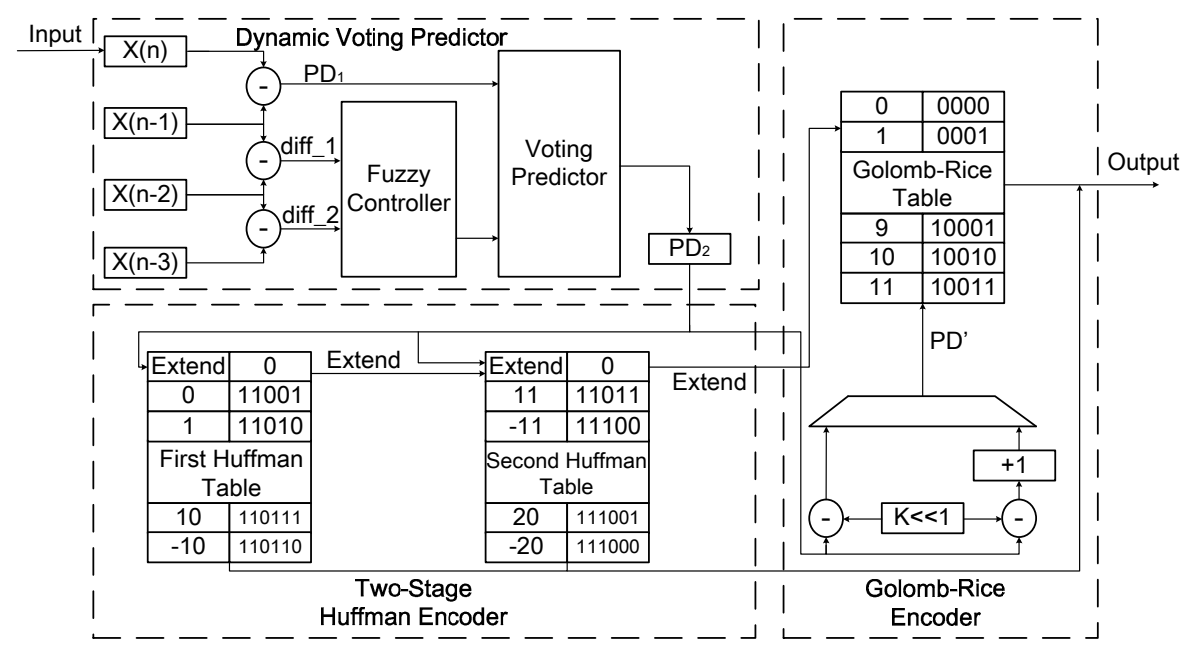

Figure 5. VLSI architecture of the proposed lossless EEG compression algorithm. 


\subsubsection{Dynamic Voting Predictor}

The predictor component in Figure 5 illustrates that the voting predictor is composed of five registers, three subtractors, one fuzzy controller, and one voting predictor. The four registers in the input stage are used to store the input data $X(n), X(n-1), X(n-2)$ and $X(n-3)$. The three subtractors provide the values for $P D_{1}$, diff_1, and diff_2. Both diff_1 and diff_2 are used as inputs to the fuzzy controller. Their output together with $P D_{1}$ are used as inputs to the voting predictor. The fifth register $P D_{2}$ holds the final output value of the adaptive voting predictor. Register $P D_{2}$ is interconnected to both of the two-stage Huffman and Golomb-Rice encoders and is also used as the pipeline register to improve the performance of the proposed design.

\subsubsection{Entropy Encoder}

The entropy encoder is composed of both the two-stage Huffman and Golomb-Rice components as shown in Figure 5. In the two-stage Huffman encoder component, two Huffman tables were used as look-up tables. The two-stage Huffman encoder checks the value of $P D_{2}$ which stores the output of the adaptive voting predictor. If the value is within the encoding range of the two-stage Huffman encoder, the value of $P D_{2}$ will be encoded using the two-stage Huffman encoder and will be directly sent as the final output. Otherwise, the value of $P D_{2}$ will be passed through a pre-processing stage where Equation (1) is utilized. The pre-processing procedure ensures that the data will be passed on to the Golomb-Rice encoder through a multiplexer, which has a range of values that will better fit what is required by the Golomb-Rice look-up table. The resulting data of the Golomb-Rice encoder is of a variable length code that will be the output of the proposed lossless EEG controller that is ready for transmission.

\subsection{Simulation and Dataset}

The CHB-MIT Scalp EEG Database [25] was utilized to examine the proposed algorithm in terms of its compression rate. The standard EEG pattern is recorded in European Data Format (edf) containing one uninterrupted digitized polygraphic recording. The original EEG signals in 23 channels were sampled at 256 samples per second with 16-bit resolution and 1 hour duration. A software program was used to extract the information from the edf file as well as to restore the original EEG pattern. The extracted data from the edf file was compressed into a bitstream of data by using the different prediction and entropy encoders. The resulting encoded bitstream of data was decoded to verify that it has the same data as that of the original data extracted from the edf file. The compression rates were computed by getting the ratio of the size of the original data and the size of the encoded bitstream.

The proposed lossless EEG encoding algorithm was implemented using Verilog code and was simulated using the ncverilog tool. The VLSI architecture was synthesized using a design compiler with a TSMC $0.18 \mu \mathrm{m}$ CMOS generic logic process technology.

\section{Results and Discussion}

Table 2 lists the compression rates for all four functions: linear, slope, adaptive trending and the proposed fuzzy and dynamic voting predictors operating with Huffman and Golomb-Rice encoders. The compression rates of the EEG signals are presented using the average compression rate using all 23 channels of the EEG signals. It is apparent that the voting prediction with entropy coding design proposed in this study has significantly improved the compression rates. Moreover, it drastically decreased the entropy coding size. The design of the prediction methodology is crucial since the prediction optimizes the frequency distribution of the values by making the values closer to a certain value that is usually 0 . In addition, the prediction minimizes the absolute values which significantly improves the performance of the entropy coding by assigning the length of the codes according to the values of the input data. 
Table 2. Compression rates of different prediction methodologies for 23 channels of CHB-MIT Scalp EEG Database.

\begin{tabular}{ccccc}
\hline Channels & Linear [6] & Slope [18] & Adaptive Trending [20] & This Study \\
\hline 1 & 1.72 & 1.69 & 1.73 & 1.98 \\
2 & 2.00 & 1.88 & 1.96 & 2.27 \\
3 & 2.01 & 1.89 & 1.97 & 2.31 \\
4 & 2.32 & 2.13 & 2.27 & 2.65 \\
5 & 1.64 & 1.63 & 1.67 & 1.87 \\
6 & 2.14 & 2.07 & 2.15 & 2.48 \\
7 & 2.49 & 2.35 & 2.43 & 2.74 \\
8 & 2.21 & 2.08 & 2.21 & 2.56 \\
9 & 1.72 & 1.73 & 1.76 & 1.96 \\
10 & 2.36 & 2.27 & 2.35 & 2.67 \\
11 & 2.49 & 2.38 & 2.47 & 2.74 \\
12 & 2.00 & 1.83 & 1.97 & 2.44 \\
13 & 1.73 & 1.66 & 1.74 & 2.02 \\
14 & 1.85 & 1.75 & 1.84 & 2.18 \\
15 & 2.07 & 1.94 & 2.03 & 2.38 \\
16 & 1.91 & 1.79 & 1.92 & 2.35 \\
17 & 2.65 & 2.54 & 2.60 & 2.86 \\
18 & 2.68 & 2.58 & 2.63 & 2.87 \\
19 & 2.01 & 1.89 & 1.97 & 2.31 \\
20 & 1.87 & 1.77 & 1.87 & 2.18 \\
21 & 1.93 & 1.92 & 1.96 & 2.26 \\
22 & 2.08 & 1.95 & 2.04 & 2.40 \\
23 & 2.07 & 1.94 & 2.03 & 2.38 \\
Average & 2.05 & 1.95 & 2.04 & 2.35 \\
\hline
\end{tabular}

Table 3 shows the specification of the area, power and the gate-count of the previous lossless compression designs in $[6,17]$ and this work. The advantage of the proposed design in terms of area is the use of a combinational logic that occupied most of the cell area which resulted to a reduction in the hardware cost to at least $37.3 \%$ compared to previous designs. The proposed design of this work has a total power consumption of $2.5867 \mathrm{~mW}$ at a global operating voltage of $1.62 \mathrm{~V}$ and a clock frequency of $100 \mathrm{MHz}$. Even though the combinational logic occupied most of the cell area, it consumed only one-tenth of the power with the registers consuming $89.26 \%$. Based on the results listed in Table 4, a limitation of the proposed design is its power consumption. Thus, minimizing the power consumed by the registers is a major concern in future improvement.

Looking into the cell report of this design, the gate count resulted to 8405 NAND-equivalent gates for the entire design with the voting prediction component contributing $68.48 \%$ of the total gate count. This is mainly due to the fact that the voting prediction component is the most complex step in the compression algorithm. Previous studies in $[6,17]$ showed a gate count of 13,400 and 53,900, respectively. Compared with the previous studies, this work showed a $37.3 \%$ reduction in terms of gate count. Moreover, this work showed an $84.4 \%$ reduction in hardware cost.

The proposed lossless EEG encoding algorithm was tested using the CHB-MIT Scalp EEG Database and conceded an average of 2.35 compression rate. Previous studies in $[6,17]$ showed an average compression rate of 2.05 and 1.37, respectively. This work showed a $14.6 \%$ and $58.30 \%$ increase in the compression rate compared to [6,17], respectively. The performance of the proposed VLSI architecture was also verified. Synthesis results recorded that the submitted encoder design contains 8405 NAND-equivalent gate counts, which is $37.3 \%$ and $84.4 \%$ less than the previous studies in [6,12], respectively. The core area in this design is $578,000 \mu^{2}$ while its power consumption is $2.58 \mathrm{~mW}$ operating at $100 \mathrm{MHz}$. The power saving of this study reaches $54.77 \%$ which is better than $51.21 \%$ and $27 \%$ in previous studies in $[6,17]$, respectively. 
Table 3. Performance comparison of the proposed lossless EEG encoding algorithm and its VLSI architecture with a previous work.

\begin{tabular}{cccc}
\hline Performance Parameters & Chen et al. [6] & Chua et al. [17] & This Work \\
\hline Compression Rate & 2.05 & 1.37 & 2.35 \\
Process $(\mu \mathrm{m})$ & 0.1 & 0.065 & 0.18 \\
Frequency $(\mathrm{MHz})$ & 100 & 24 & 100 \\
Voltage $(\mathrm{V})$ & 1.80 & 1.00 & 1.80 \\
Gate Count $(\mathrm{k})$ & 13.4 & 53.9 & 8.4 \\
Area $\left({\mathrm{k} \mu \mathrm{m}^{2}}^{)}\right.$ & 134 & 58 & 84 \\
Normalized Area & 1.59 & 5.29 & 1.00 \\
Power Save $(\%)$ & 51.21 & 27.00 & 54.77 \\
\hline
\end{tabular}

Table 4 lists the energy savings of the original data and the compressed data using both the Bluetooth and Zigbee wireless transmitters. The Ecomp is derived using the power consumption with a clock frequency of $100 \mathrm{MHz}$ for the input data rate used in circuit simulation per bit divided. In addition, the power has a high-positive correlation to the amount of data. Consequently, the power consumption is reduced relative to the compression rate. The Etx_original and Etotal_original are the energy and the total energy of the original data which are raw and uncompressed. Using the $C R$ value of 2.35, the energy after compression is computed as Etx_CR and Etotal_CR. It is clearly shown that the amount of data is reduced 2.35 times by $C R$ and thus the energy is significantly. The results show that even though this design consumes more power than previous designs, its energy savings using wireless transmitters can be improved.

Table 4. Energy saving for Bluetooth and Zigbee wireless transmitters.

\begin{tabular}{ccc}
\hline Performance Parameters & Bluetooth & Zigbee \\
\hline Etx_original (nJ/bit) & 143 & 296 \\
Etotal_original (nJ/bit) & 143 & 296 \\
Compression Rate & 2.35 & 2.35 \\
Etx_CR (nJ/bit) & 60.85 & 125.96 \\
Ecomp (nJ/bit) & 0.005 & 0.005 \\
Etotal_CR (nJ/bit) & 60.855 & 125.965 \\
Energy Savings (\%) & 57.44 & 57.44 \\
\hline
\end{tabular}

\section{Conclusions}

This paper presented a novel lossless, high-compression-rate with a low-complexity EEG compression algorithm that is based on a fuzzy decision, dynamic voting prediction and tri-stage entropy coding. The proposed VLSI design had significantly increased the compression rate to $14.6 \%$ and significantly reduced the hardware cost to $37.3 \%$ compared to previously reported designs in literatures. A limitation of the proposed design is its power consumption which can be reduced by improving the power consumption of the registers for future works. The proposed design is suitable for applications in WBAN, WBSN, wearable devices, as well as in Internet of Things (IoT) devices.

Author Contributions: Conceptualization, C.-A.C., C.W. and S.-L.C.; Software, C.W. and S.-L.C.; Validation, P.A.R.A. and C.-A.C.; Formal Analysis, C.-A.C., C.W., P.A.R.A. and S.-L.C.; Investigation, C.-A.C., C.W. and S.-L.C.; Resources, C.-A.C. and C.W.; Data Curation, C.-A.C., C.W., P.A.R.A. and S.-L.C.; Writing-Original Draft Preparation, C.W. and C.-A.C.; Writing-Review \& Editing, C.-A.C., P.A.R.A. and S.-L.C.; Visualization, C.-A.C., P.A.R.A. and S.-L.C.; Supervision, S.-L.C.; Project Administration, C.-A.C. and S.-L.C.; Funding Acquisition, C.-A.C. and S.-L.C.

Funding: This work was supported by the Ministry of Science and Technology (MOST), Taiwan, under grant numbers of MOST-107-2218-E-131-002, MOST-107-2221-E-033-057, MOST-107-2622-E-131-007-CC3, MOST-106-2622-E-033-014-CC2, MOST-106-2221-E-033-072, MOST-106-2119-M-033-001, and the National Chip Implementation Center, Taiwan.

Conflicts of Interest: The authors declare no conflict of interest. 


\section{References}

1. Nguyen, T.H.; Yang, D.L.; Chung, W.Y. A high-rate BCI speller based on eye-closed EEG signal. IEEE Access 2018, 6, 33995-34003. [CrossRef]

2. Liew, S.H.; Choo, Y.H.; Low, Y.F.; Yusoh, Z.I.M. EEG-based biometric authentication modelling using incremental fuzzy-rough nearest neighbour technique. IET Biom. 2018, 7, 145-152. [CrossRef]

3. Wen, T.; Zhang, Z. Deep convolution neural network and autoencoders-based unsupervised feature learning of EEG signals. IEEE Access 2018, 6, 25399-25410. [CrossRef]

4. Giorgi, G. A combined approach for real-time data compression in wireless body sensor networks. IEEE Sens. J. 2017, 17, 6129-6135. [CrossRef]

5. Chen, S.L.; Tuan, M.C.; Lee, H.Y.; Lin, T.L. VLSI implementation of a cost-efficient micro control unit with an asymmetric encryption for wireless body sensor networks. IEEE Access 2017, 5, 4077-4086. [CrossRef]

6. Chen, S.L.; Lee, H.Y.; Chen, C.A.; Huang, H.Y.; Luo, C.H. Wireless body sensor network with adaptive low power design for biometrics and healthcare applications. IEEE Syst. J. 2009, 3, 398-409. [CrossRef]

7. Salleh, S.M.; Jusoh, H.; Ismail, A.H.; Kamarudin, M.R.; Norbles, P.; Rahim, M.K.; Sabapathy, T.; Osman, M.N.; Jais, M.I.; Soh, P.J. Textile antenna with simultaneous frequency and polarization reconfiguration for WBAN. IEEE Access 2018, 6, 7350-7358. [CrossRef]

8. Khan, F.A.; Haldar, N.A.H.; Ali, A.; Iftikhar, M.; Zia, T.A.; Zomaya, A.Y. A continuous change detection mechanism to identify anomalies in ECG signals for WBAN-based healthcare environments. IEEE Access 2017, 5, 13531-13544. [CrossRef]

9. Wang, L.; Hu, F.; Ling, Z.; Wang, B. Wireless information and power transfer to maximize information throughput in WBAN. IEEE Internet Things J. 2017, 4, 1663-1670. [CrossRef]

10. Peng, H.; Tian, Y.; Kurths, J.; Li, L.; Yang, Y.; Wang, D. Secure and energy-efficient data transmission system based on chaotic compressive sensing in body-to-body networks. IEEE Trans. Biomed. Circuits Syst. 2017, 11, 558-573. [CrossRef] [PubMed]

11. Hooshmand, M.; Zordan, D.; Testa, D.D.; Grisan, E.; Rossi, M. Boosting the battery life of wearables for health monitoring through the compression of biosignals. IEEE Internet Things J. 2017, 4, 1647-1662. [CrossRef]

12. Srinivasan, K.; Dauwels, J.; Reddy, M.R. Multichannel EEG compression: Wavelet-based image and volumetric coding approach. IEEE J. Biomed. Health Inf. 2013, 17, 113-120. [CrossRef] [PubMed]

13. Sriraam, N.; Eswaran, C. An adaptive error modeling scheme for the lossless compression of EEG signals. IEEE Trans. Inf. Technol. Biomed. 2008, 12, 587-594. [CrossRef] [PubMed]

14. Shaw, L.; Rahman, D.; Routray, A. Highly efficient compression algorithms for multichannel EEG. IEEE Trans. Neural Syst. Rehabil. Eng. 2018, 26, 957-968. [CrossRef] [PubMed]

15. Alvarez, G.D.; Favaro, F.; Lecumberry, F.; Martin, A.; Oliver, J.P.; Oreggioni, J.; Ramirez, I.; Seroussi, G.; Steinfeld, L. Wireless EEG system achieving high throughput and reduced energy consumption through lossless and near-lossless compression. IEEE Trans. Biomed. Circuits Syst. 2018, 12, 231-241. [CrossRef] [PubMed]

16. Zhou, P.Y.; Chan, K.C.C. Fuzzy feature extraction for multichannel EEG classification. IEEE Trans. Cogn. Dev. Syst. 2016, 10, 267-279. [CrossRef]

17. Chua, E.; Fang, W. Mixed bio-signal lossless data compressor for portable brain-heart monitoring systems. IEEE Trans. Consum. Electron. 2011, 57, 267-273. [CrossRef]

18. Chen, C.A.; Chen, S.L.; Huang, H.Y.; Luo, C.H. An efficient micro control unit with a reconfigurable filter design for wireless body sensor networks (WBSNs). Sensors 2012, 12, 16211-16227. [CrossRef] [PubMed]

19. Chen, S.L.; Wang, J.G. VLSI implementation of a low-power cost-efficient lossless ECG encoder design for wireless healthcare monitoring application. Electron. Lett. 2013, 49, 91-93. [CrossRef]

20. Chen, S.L.; Luo, G.A.; Lin, T.L. Efficient fuzzy-controlled and hybrid entropy coding strategy lossless ECG encoder VLSI design for wireless body sensor networks. Electron. Lett. 2013, 49, 1058-1060. [CrossRef]

21. Chen, S.L. A power-efficient adaptive fuzzy resolution control system for wireless body sensor networks. IEEE Access 2015, 3, 743-751. [CrossRef]

22. Chen, S.L.; Villaverde, J.F.; Lee, H.Y.; Chung, W.Y.; Lin, T.L.; Tseng, C.H.; Lo, K.A. A power-efficient mixed-signal smart ADC design with adaptive resolution and variable sampling rate for low-power applications. IEEE Sens. J. 2017, 11, 3461-3469. [CrossRef] 
23. Chen, C.A.; Chen, S.L.; Huang, H.Y.; Luo, C.H. An asynchronous multi sensor micro control unit for wireless body sensor networks (WBSNs). Sensors 2011, 11, 7022-7036. [CrossRef] [PubMed]

24. Moody, G.B.; Mark, R.G. The impact of the MIT/BIH arrhythmia database. IEEE Eng. Med. Biol. Mag. 2001, 20, 45-50. [CrossRef] [PubMed]

25. Goldberger, A.L.; Amaral, L.; Glass, L.; Hausdorff, J.M.; Ivanov, P.; Mark, R.G.; Mietus, J.E.; Moody, G.B.; Peng, C.K.; Stanley, H.E. PhysioBank, PhysioToolkit, and PhysioNet: Components of a New Research Resource for Complex Physiologic Signals. Circulation. Available online: http:/ / circ.ahajournals.org/cgi/ content/full/101/23/e215 (accessed on 15 July 2018).

2018 by the authors. Licensee MDPI, Basel, Switzerland. This article is an open access article distributed under the terms and conditions of the Creative Commons Attribution (CC BY) license (http:/ / creativecommons.org/licenses/by/4.0/). 